\title{
Zwölf Thesen zum Thema Zeitwohlstand
}

\author{
Spricht man von Zeitwohlstand, mag man \\ intuitiv an einen Zugewinn an Freizeit denken. \\ Doch dies ist nur ein Aspekt. Mit unseren \\ Thesen wollen wir die Vielschichtigkeit dieser \\ Erzählung nachzeichnen. \\ Von Gerrit von Jorck, Maren Birkenstock, \\ Richard Harnisch und Sebastian Stagl
}

\footnotetext{
D
} ie fünfte Sommerakademie der Vereinigung für ökologische Wirtschaftsforschung (VÖW) lud zu einer „Zeitwerkstatt" ein. Dort haben wir uns auf die Suche nach einem neuen gesellschaftlichen Narrativ gemacht: dem Zeitwohlstand und seiner sozial-ökologischen Dimension. Mit den zwölf folgenden Thesen wollen wir uns diesem schillernden Begriff nähern und zur Diskussion einladen.

Zeitgerechtigkeit durch soziale Sicherung. Verfügbare Zeit kann nur Zeitwohlstand erzeugen, wenn Existenzängste beseitigt und Grundbedürfnisse befriedigt sind. Soziale Absicherung ist daher eine Grundvoraussetzung für Zeitwohlstand. Um diesen für alle zu ermöglichen und nicht nur als Privileg für jene, die es sich zufällig leisten können, muss es auch materielle Umverteilung geben und in diesem Sinne mehr Zeitgerechtigkeit geschaffen werden.

Wettbewerbsgesellschaft. Wettbewerb ist zum bestimmenden Treiber gesellschaftlicher Entwicklung geworden und beschleunigt das Lebenstempo. Dabei kommt es zur Herausbildung fiktiver Waren wie Arbeit und Natur. Die menschliche Existenz wird in Abhängigkeit des Arbeitsmarktes gestellt, ob nun direkt über den Verkauf der eigenen Arbeitskraft oder indirekt über ein Sozialstaatssystem, welches die Kommodifizierung der Arbeit forciert und dessen Finanzierung vom Arbeitsmarkt abhängt. Hierdurch entsteht eine Spirale der Beschleunigung.

Gute Arbeit. Durch eine Verkürzung der Arbeitszeit kann mehr freie Zeit entstehen. Aber Zeitwohlstand hat in Zusammenhang mit Arbeit auch eine qualitative Dimension: die Durchsetzung von sogenannter "Guter Arbeit“ im Sinne von Zeitsouveränität, gekennzeichnet durch Entschleunigung, mehr Mitbestimmung über Zeiträume und Arbeitsmengen, Anpassung des Arbeitslebens an die Bedürfnisse der Arbeitenden. Die Dichotomie aus Arbeitszeit und Freizeit bleibt dabei zwar erhalten, die Trennung zwischen dem Reich der Freiheit und dem Reich der Notwendigkeit wird jedoch dadurch aufgehoben.

Produktivität und technologischer Fortschritt. Eine Steigerung der Produktivität könnte zu mehr Zeitwohlstand führen. Dies ist in dem Maße möglich, in dem immer weniger Lebenszeit für mühevolle Produktion geopfert werden muss. Ein problematischer Rebound-Effekt entsteht allerdings dann, wenn die eingesparte Zeit genutzt wird, um noch mehr zu arbeiten. Auch Subsistenzarbeit ist in diesem Zusammenhang ambivalent. Sie kann je nach ihrem Ausmaß zu Zeitwohlstand, aber auch zu dessen Gegenteil, Zeitarmut, beitragen.

Imperiale Lebensweise. Die beschleunigte imperiale Lebensweise des Globalen Nordens radikalisiert sich im Globalen Süden und vermindert dadurch den Zeitwohlstand auch jenseits westlicher Wohlstandsgesellschaften. In Indien etwa leidet die Mittelschicht unter extremer Zeitarmut. Ihr Leistungsethos speist sich aus permanenter Angst vor dem sozialen Absturz. Hier können Post-Development-Bewegungen wie Buen Vivir Gegenentwürfe zur beschleunigten Lebensweise aufzeigen.

Suffizienz. Die Gewohnheit und der Zwang, ständig zu konsumieren, sei dies durch sogenannten Statuskonsum oder schlicht durch den Mangel an konsumfreien öffentlichen Räumen, führen zu einem Work-and-Spend-Cycle. Mehr auf Zeitwohlstand als auf materiellen Wohlstand zu setzen, könnte hier einen Ausweg bedeuten: Wenn weniger an Zeitarmut gelitten wird, könnte der Drang, durch Konsum eine Kompensation zur häufig entfremdeten Erwerbsarbeit zu schaffen, gesenkt werden. An diese Stelle tritt ein Zugewinn an freier Zeit.

Zeitautonomie. Zeitwohlstand bedeutet Zeitautonomie und damit die Befreiung von Fremdbestimmung und Bevormundung. Das ist jedoch nicht gleichzusetzen mit der Abwesenheit von Bindungen und Verpflichtungen. Im Gegenteil besteht Zeitwohlstand in frei gewählten Abhängigkeiten, die durchaus langfristig sein können. Zeitwohlstand braucht eine individuelle Balance zwischen Zeitautonomie und dem Vertrauen-Können in zuverlässige und verbindliche Abhängigkeiten.

Multioptionsgesellschaft. Wir leben heutzutage in einer Multioptionsgesellschaft, mit stets vielen und immer mehr Möglichkeiten, die Zeit zu verbringen. Mehr Optionen als wir wahrnehmen können. Wenn die Realisierung einzelner Optionen jedoch als Zwang erlebt wird, kann Zeitwohlstand in Gefahr geraten: Die Zeit verdichtet sich und dies führt zu einem unausgewogenen Verhältnis von Zeiten des Erlebens und Zeiten der Reflexion. Das Ergebnis kann trotz Zeitsouveränität Zeitarmut sein. 
Reflexionszeiten. Die Herausforderungen der multiplen Krise erfordern Zeiten der Reflexion in sämtlichen gesellschaftlichen Bereichen. Je weiter das Eingreifen der Menschen in ihre Umwelten und das Begreifen der Folgen auseinanderklaffen, desto wichtiger wird Zeitwohlstand als Voraussetzung für Reflexion. Auch auf individueller Ebene ist ausreichend Zeit für ein Reflektieren des eigenen Lebensentwurfs und der eigenen Willensbildung notwendig, um durch eigenes Handeln Zeitwohlstand $\mathrm{zu}$ realisieren.

Selbststeuerung und Entschleunigung. Die Anforderung, in einer komplexer werdenden Umwelt sozial und ökonomisch zu bestehen, überfordert viele Menschen und macht sie krank. In dieser Umwelt besteht der Zwang, Selbststeuerungsfähigkeiten permanent weiterzuentwickeln. Das unternehmerische Selbst muss erfolgreich vermarktet und verwaltet werden. Um in einer solchen Welt dennoch Zeitwohlstand zu erfahren, ist die Entwicklung zivilisatorischer Entschleunigungsfähigkeiten gefragt. Es geht um die Kunst, sein eigenes Tempo zu finden und sich in diesem mit der Umwelt und mit anderen zu synchronisieren, Mußezeit wiederzuentdecken und wertzuschätzen.

Muße. Zeitwohlstand besteht dann, wenn die Möglichkeit gegeben ist, sich der Muße, Kontemplation und Entschleunigung hinzugeben. Dies kann auch heißen, sich bewusst treiben $\mathrm{zu}$ lassen und neuen Reichtum an Erfahrungen zu gewinnen. Elemente dieses Reichtums wären zum Beispiel das Dérive der Situationisten, die den Zufall das Leben bestimmen lassen; das Sich-Zeit-Nehmen für Experimente, der Hedonismus der Überraschungen oder eine Kultur des Feierns. Nicht ein Mehr an Konsumieren stünde hier im Vordergrund, sondern ein Mehr an Erleben.

Synchronisation und Resonanz. Eine Voraussetzung für Zeitwohlstand ist das Vermögen, sich auf mehreren Ebenen zu synchronisieren. Erstens betrifft dies die Synchronisation mit der natürlichen Umwelt. Die Naturressourcen sollten unter Berücksichtigung der Regenerativität der Natur genutzt werden. Eine zweite Voraussetzung ist die innergesellschaftliche Synchronisation beziehungsweise die Herstellung von Reziprozität mit der Mitwelt und drittens geht es um die individuelle Synchronisation, also die Suche nach dem eigenen Rhythmus im Denken und Handeln. Vor diesem Hintergrund bedeutet das Streben nach Zeitwohlstand, Synchronisationskünstler auf mehreren Ebenen zu sein. Synchronisation ist dabei Voraussetzung für Resonanzerfahrungen, sprich mit sich und seiner Umwelt im Zusammenklang zu stehen.

\section{AUTOREN + KONTAKT}

Die Zeitpioniere sind eine Arbeitsgruppe der Vereinigung für ökologische Wirtschaftsforschung (VÖW) Maren Birkenstock, Richard Harnisch, Gerrit von Jorck und Sebastian Stagl bilden das Redaktionsteam der Arbeitsgruppe. Die zwölf Thesen sind aus der VÖW-Sommerakademie „Zeitwerkstatt zur sozial-ökologischen Dimension von Zeitwohlstand“ hervorgegangen.

Vereinigung für ökologische Wirtschaftsforschung (VÖW), Potsdamer Str. 105, 10785 Berlin. Tel.: +49 30 884594-0, E-Mail: maren.birkenstock@posteo.de, richard.harnisch@ioew.de, gerrit.jorck@ioew.de sebastian.stagl@gmail.com
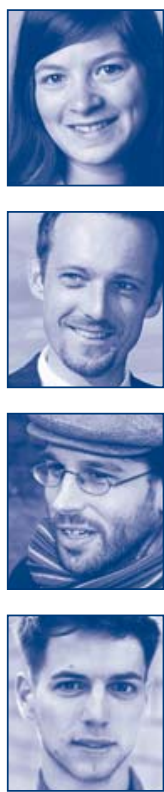

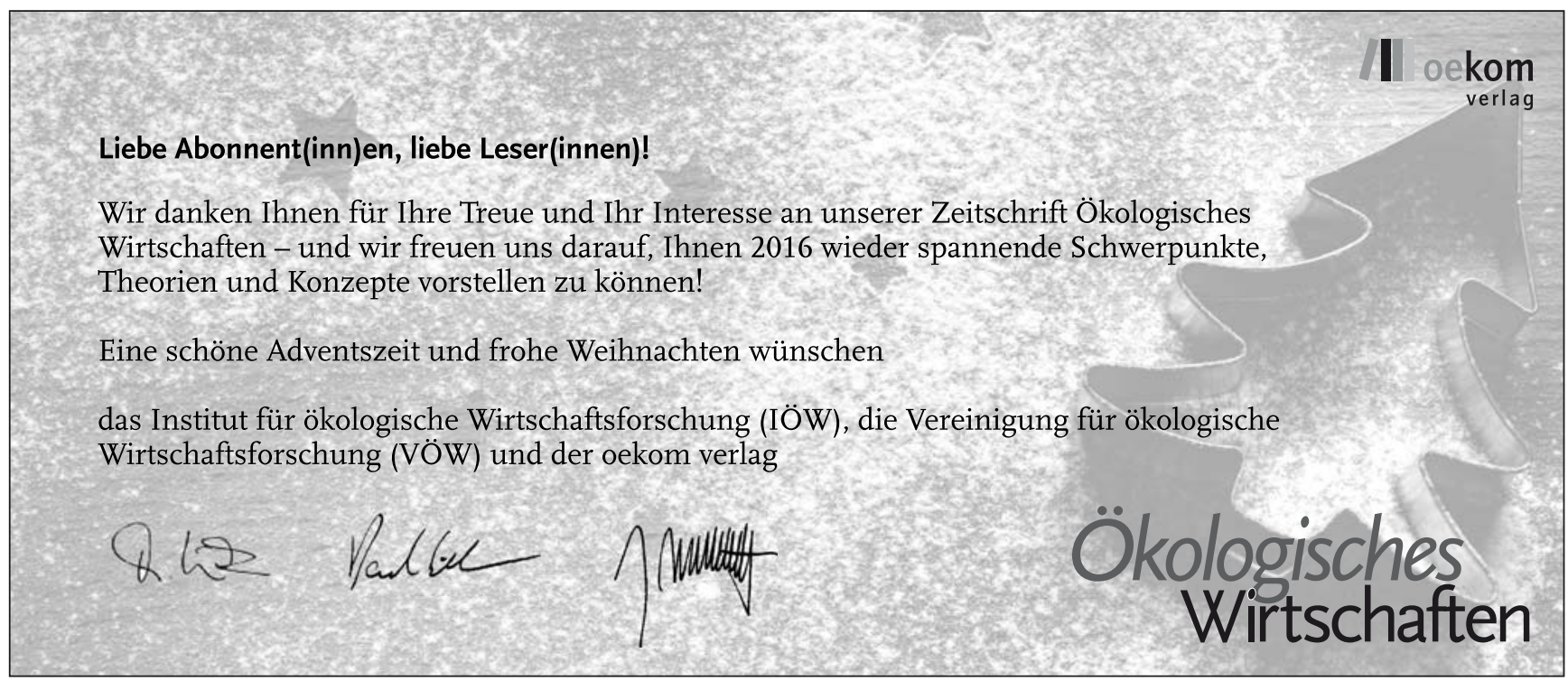

